\title{
Efficacy of single dose antihistamine vs. single dose valerian-hops in subjective sleep measures among war refugees: a comparison trial
}

\author{
Omar Salem Gammoh ${ }^{1}$, Ahmed Al-Smadi¹, Tareq Mukattash², Waleed Al-Katib1, Hrayr Attarian² \\ Mohammad AL-ShaWAGFEH ${ }^{4}$
}

${ }^{1}$ American University of Madaba, Madaba, Jordan.

2 Jordan University of Science and Technology, Jordan.

${ }^{3}$ Northwestern University Feinberg School of Medicine.

${ }^{4}$ King Hussain Hospital, Royal Medical Services.

Received: 7/29/2016 - Accepted: 1/16/2017

DOl: 10.1590/0101-60830000000114

\begin{abstract}
Background: Many sedatives and anxiolytics are used in single dose or chronically to aid sleep. Clinically important sedatives include valerian-hops and antihistamines as they are used over the counter and are highly accessible and safe agents. Objectives: To evaluate and compare a single dose of chlorpheniramine versus valerian-hops combination in modulating subjective sleep measures in insomniac war refugees. Methods: Insomnia among refugees was screened using the Insomnia Severity Index (ISI). Insomniac subjects were randomized to received a single dose valerian-hops $(320 / 80 \mathrm{mg})(\mathrm{n}=65)$, or chlorpheneramine $(4 \mathrm{mg})(\mathrm{n}=50)$ or placebo $(\mathrm{n}=76)$ two hours prior sleeping. Participants were instructed to complete Leeds Sleep Evaluation Questionnaire (LSEQ), visual analogue scales of anxiety and sedation. Also sleep latency, total hours slept and self-rated improvement were obtained. Results: Almost $75 \%$ of screened refugees had insomnia. Chlorpheneramine reduced sleep latency and anxiety significantly, however it resulted in poor sleep quality. Valerian-hops group showed marked anxiolysis one hour after dosing, a sleep quality similar to placebo and better than chlorpheneramine, and better alertness compared to placebo. Participants satisfaction was higher with chlorpheneramine and there was no difference in the total hours slept. Discussion: Valerian-hops combination may provide better sleep quality than antihistamines.
\end{abstract}

Gammoh OS et al. / Arch Clin Psychiatry. 2017;44(2):35-9

Keywords: Valerian, antihistamines, insomnia, refugees.

\section{Introduction}

Insomnia is a primary sleep disorder whereby patients have difficulties falling asleep, with maintaining sleep during the night, or with early wakening ${ }^{1}$.

It is estimated that more than $30 \%$ of the populations of industrialized countries report sleep disturbances ${ }^{2,3}$. Improper sleep has a negative impact on one's health-related quality of life and impairs patients' social, physical and cognitive functioning4.

According to the 2012 report of the United Nations High Commissioner for Refugees (UNHCR), there were 15.4 million refugees worldwides.

Refugees are highly predisposed to developing neurological and psychological disorders such as anxiety, depression and posttraumatic stress disorder ${ }^{6,7}$.

Surprisingly, insomnia prevalence and treatment is seldom studied in refugee populations. Two recent studies reported insomnia prevalence of $38 \%$ and $44 \%$ among displaced people 6,8 .

Benzodiazepines and sedating antihistamines are among the most prescribed sedative hypnotics for both chronic use or when needed as a single dose 9 .

Although these synthetic medications have proved their efficacy in sleep induction, they are associated with side effects such as dizziness, headache, dependence and tolerance ${ }^{10,11}$. Therefore, alternative therapeutic options such as valerian root have increased in popularity.

Valeriana officinalis is a hardy perennial flowering plant ${ }^{12}$. It is native to Europe and parts of Asia and has been naturalized in North America for commercial use. Valerian root is formulated as tablets or soft gelatin capsules ${ }^{13}$ and is typically administered orally to treat mild insomnia and anxiety in combination with hops. It is assumed to activate GABA through Valerenic acid as the active ingredient ${ }^{13}$.

Comparative trials between valerian-hops and synthetic sedatives/anxiolytics are rare. One such study compared the efficacy of a valerian-hops combination to that of diphenhydramine in insomnia over 6 weeks. They revealed modest improvement in sleep outcome measurements in both treatment arms ${ }^{14}$. However, no previous studies have compared the efficacy of single doses of valerian-hops to sedating antihistamine in terms of subjective sleep measures.

Therefore, the objective of the current study is to evaluate and compare the effect of a single dose of valerian-hops against a single dose of a widely used antihistamine, chlorpheniramine, when modulating subjective sleep measures among refugees with insomnia. Subjective sleep parameters were evaluated according to Leeds Sleep Evaluation Questionnaire (LSEQ). Other studied parameters were: sleep latency, sleeping hours, sedation, anxiety and self-rated clinical evaluation.

\section{Methods}

\section{Study design and outcome measures}

Initially, refugees were screened for insomnia clinically and by using the Insomnia Severity Index (ISI). Afterwards, insomniac refugees were randomly assigned to receive a single dose of valerian-hops, chlorpheniramine or a placebo. The outcome measures were difference in sleep quality according to the (LSEQ), anxiety, sedation, sleep latency, actual hours slept and self-rated clinical improvement. Ethical approval was obtained from the institutional review board (IRB) at King Hussain Hospital. Potential participants were provided with details on the study and had to sign a detailed IRB approved consent form prior to participation. Each participant was informed about his/her right to withdraw from the study at any time.

\section{Sample and sampling method}

Adult refugees living in two cities in Jordan (Amman and Mafraq) were approached during their visit to the Caritas Medical Centre. 
At the screening phase, refugees with a prior history of psychological or mental illnesses and ones using anxiolytics or antidepressants or any drugs affecting the central nervous system were excluded. Pregnant or lactating females were also excluded.

\section{Intervention}

After screening completion, insomniac refugees were asked about their willingness to participate in the trial. Willing participants were randomly assigned to receive a Cirkulin ${ }^{\oplus}$ Valerian-hops combination (320 mg of valerian root dry extract $+80 \mathrm{mg}$ of hop stabile dry extract), chlorpheniramine $4 \mathrm{mg}$ or a placebo. Randomization was performed by sequencing patients entering to the physician by using numbered closed envelopes. The prescribing physicians asked all the participants to take the single dose two-hour prior to their bed time.

Each participant was asked to complete an LSEQ at awakening. Sedation and anxiety visual analogue scales were filled one hour after the dose, after awakening and 24 hours after dose administration. Data collection and entry was performed by independent researchers blind to the interventions.

\section{Sample size calculations}

For the purpose to determine the number of participants need in each study groups, statistical $G$ power calculation was used and revealed the need for at least 44 participants in each group; this was based on 0.07 Eta Squared, and power of 0.80 . However, the authors decided to include as much as possible participants equal or greater than 44 in each group.

\section{Study instruments}

In addition to demographical and clinical details, the Arabic versions of ISI, LSEQ, the anxiety visual analogy scales, the sedation visual analogue scale, and the treatment evaluation were employed.

The ISI was developed by Morin ${ }^{15}$ and consists of seven questions with Likert type choices ranging from 0 to 4 ; a higher score indicates more sleeping problems. The total possible score for each participant ranged from 0 to 28 . Based on a previous literature participants, scoring 10 or more were considered to be insomniacs ${ }^{16}$. Prior research utilized the Arabic version of ISI, which was showed to be reliable with an internal consistency of $0.84^{17}$. In the current study, ISI was showed to have good reliability with a Cronbach's alpha score of 0.89 .
The LSEQ was used to assess sleeping patterns among refugees ${ }^{18}$ The scale was self-reporting and consisted of ten $100-\mathrm{mm}$ visual analogue types of questions, which measure four dimensions of sleep: ease of getting to sleep (GTS) three questions, quality of sleep (QOS) two questions, awakening from sleep (AFS) two questions, and behavior following wakefulness (BFW) three questions. Each participant responded by marking each visual analogue line from 0 to $100 \mathrm{~mm}$. The mark position indicated the changes that occur in sleeping; marks closer to the left indicate improvement, closer to the right indicate impairment, and closer to the middle indicates no changes. The LSEQ was translated to the Arabic language using the standardized back translation method, and each factor showed good reliability with Cronbach's alpha scores ranging from 0.90 to 0.94 .

In addition to these measures, a visual analogue scale for each anxiety and sedation that ranged from 0 to 10 was used. The higher score indicates higher anxiety and sedation. Moreover, at 24 hours each participant was asked to mention the minutes they needed to sleep, and the hours of sleeping during that night. Additionally, participants were asked for asked for treatment evaluation, which ranged from one to three ( 1 for no improvement, 2 for slight improvement, and 3 for marked improvement.)

\section{Statistical analysis}

All refugees' continuous data included the LSEQ, with sedation, anxiety and insomnia scores showing as normally distributed. SPSS statistical package version 21 was used to analyze the data. Descriptive statistics were used to analyze frequencies and standard deviations, means and differences in insomnia at screening level.

One-way ANOVA was used to examine the differences in the LSEQ, sedation scores, anxiety, time to sleep, and time of sleeping between the three groups (i.e. valerian-hops, chlorpheniramine and placebo). The Kruskal-Wallis test was used to examine the differences between the three groups in each treatment evaluation. The significant level was less than 0.05 for the statistical tests.

\section{Results}

A total of 373 were assessed for their eligibility. A total of 111 candidates were excluded. Therefore, two hundred sixty participants were randomized for the three study groups. A total of one hundred ninety participants successfully completed the study and their data were analyzed. Please refer to the flow chart (Figure 1).

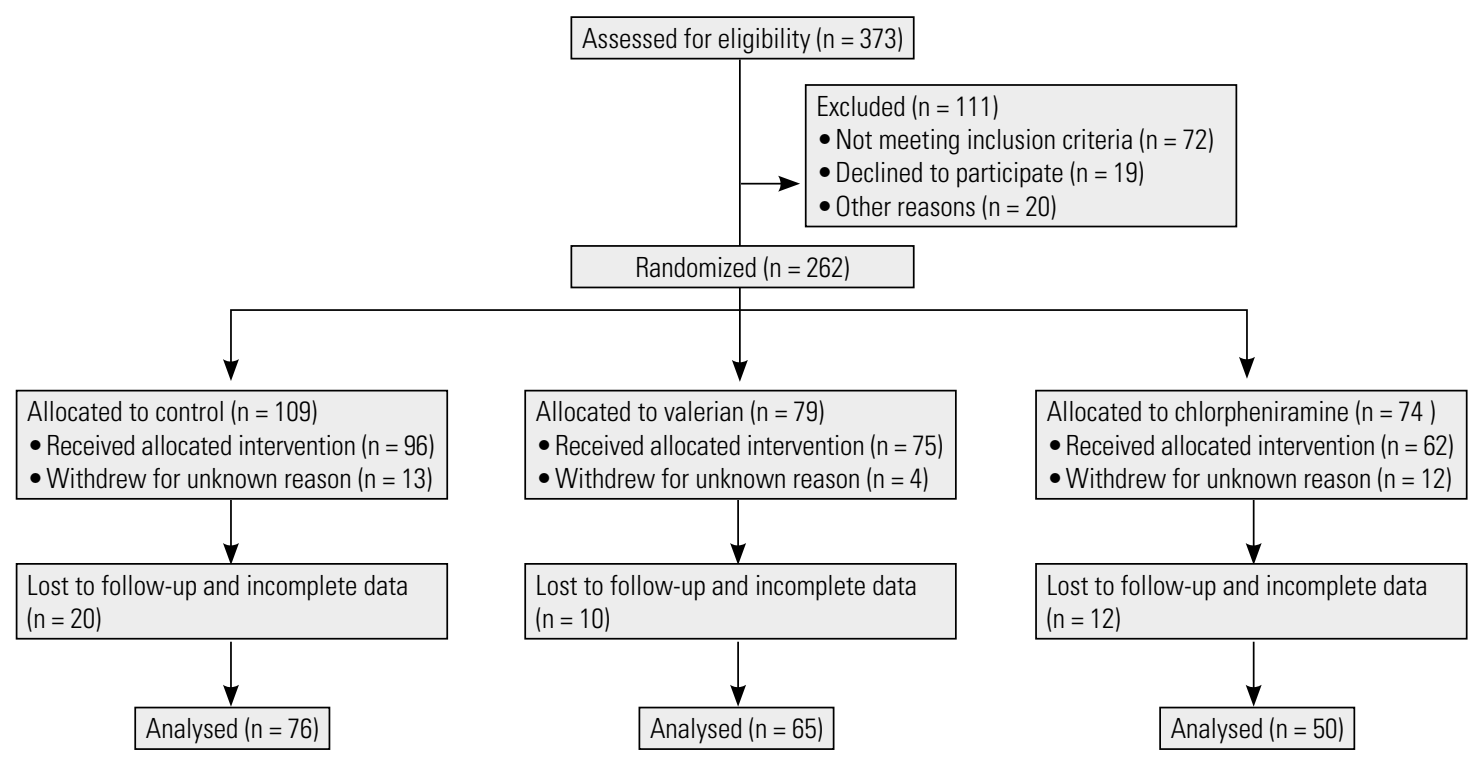

Figure 1. Study flow chart showing participants randomization. 


\section{Demographical and clinical details}

The mean age of intervention participants was $45.0(\mathrm{SD}=13.61)$. As shown in Table 1, the majority of refugees that participated in the study lived in Amman $(\mathrm{n}=124,64.9 \%)$, were female $(\mathrm{n}=$ $114,59.7 \%)$, married $(\mathrm{n}=148,77.5 \%)$, had less than a secondary school education $(\mathrm{n}=119,62.3 \%)$, were unemployed $(\mathrm{n}=114$, $59.7 \%)$, had Syrian nationality $(\mathrm{n}=131,68.6 \%)$, were non-smokers $(\mathrm{n}=113,59.2 \%)$, were not diagnosed previously with any chronic illness namely hypertension, diabetes and cardiac diseases $(\mathrm{n}=$ $110,57.6 \%)$, and had medications for chronic illnesses available (anti-hypertensives, oral hypoglycemic agents, etc.) ( $\mathrm{n}=185$, 96.9\%).

Table 1. Demographical details of intervention part of study participants

\begin{tabular}{|l|c|c|}
\hline Factors & Categories & $\begin{array}{c}\text { Number } \\
\text { (percentage) }\end{array}$ \\
\hline City & Amman & $124(64.9 \%)$ \\
\hline Gender & Mafraq & $67(35.1 \%)$ \\
\hline & Female & $114(59.7 \%)$ \\
\hline Marital status & Male & $77(40.3 \%)$ \\
\hline & Married & $148(77.5)$ \\
\hline Education level & Single & $43(22.5 \%)$ \\
\hline & Less than secondary & $119(62.3 \%)$ \\
\hline Employment & Secondary school or higher & $72(37.7 \%)$ \\
\hline & Yes & $161(84.3 \%)$ \\
\hline Smoking status & No & $30(15.7 \%)$ \\
\hline & Smoker & $78(40.8 \%)$ \\
\hline Previous chronic illness (s) & Non-smoker & $113(59.2 \%)$ \\
\hline & No & $110(57.6 \%)$ \\
\hline Medication availability & Yes & $81(42.4 \%)$ \\
\hline & No & $6(3.1 \%)$ \\
\hline Nationality & Yes & $185(96.9 \%)$ \\
\hline & Syrian & $131(68.6 \%)$ \\
\hline
\end{tabular}

\section{LSEO at awakening}

As shown in Table 2, the LSEQ was completed at awakening. One-way ANOVA test was used to examine the differences in four factors based on the type of intervention that refugees received.

The ANOVA test showed significant differences in GTS scores between the three groups $\mathrm{F}(2,189)=22.64, \mathrm{P}=0.001$, QOS scores $\mathrm{F}(2,189)=60.19, \mathrm{P}=0.001$, AFS F $(2,189)=22.55, \mathrm{P}=0.001$, and BFW F $(2,189)=28.52, \mathrm{P}=0.001$. The Scheffe post hoc test showed that the chlorpheniramine group had a significantly higher score of GTS compared to placebo $(\mathrm{p}=0.001)$ and valerian-hops $(\mathrm{p}=0.001)$. However, there were no significant differences between the placebo and valerian-hops group.

Post hoc showed that there were differences in QOS; the chlorpheniramine group had a significantly higher score of QOS compared to placebo $(\mathrm{p}=0.001)$ and valerian-hops $(\mathrm{p}=0.001)$. However, there were no significant differences between the placebo and valerian-hops groups.

In addition, the chlorpheniramine group had a significantly higher score of AFS compared to placebo $(p=0.001)$ and valerianhops $(p=0.001)$. However, there were no significant differences between the placebo and valerian-hops groups.

Moreover, valerian-hops had a significantly higher score of BFW compared to the placebo group $(\mathrm{p}=0.001)$ and lower scores compared to chlorpheniramine $(\mathrm{p}=0.001)$. In addition, the chlorpheniramine group had a significantly higher score of BFW compared to the placebo group $(\mathrm{p}=0.045)$.
Table 2. Differences in study measures based on the intervention received

\begin{tabular}{|c|c|c|c|c|}
\hline Factors & Group & $\begin{array}{c}\text { Mean } \\
\text { score at } \\
1 \text { hour }\end{array}$ & $\begin{array}{c}\text { Mean } \\
\text { score at } \\
\text { awakening }\end{array}$ & $\begin{array}{c}\text { Mean } \\
\text { score at } \\
24 \text { hours }\end{array}$ \\
\hline \multirow[t]{3}{*}{ Leeds (GTS) } & Control & & 4.82 & \\
\hline & Valerian-hops & & 5.02 & \\
\hline & Chlorpheniramine & & 6.28 & \\
\hline \multirow[t]{3}{*}{ Leeds (OOS) } & Control & & 4.85 & \\
\hline & Valerian-hops & & 5.33 & \\
\hline & Chlorpheniramine & & 6.82 & \\
\hline \multirow[t]{3}{*}{ Leeds (AFS) } & Control & & 4.79 & \\
\hline & Valerian-hops & & 5.26 & \\
\hline & Chlorpheniramine & & 6.32 & \\
\hline \multirow[t]{3}{*}{ Leeds (BFW) } & Control & & 4.80 & \\
\hline & Valerian-hops & & 5.37 & \\
\hline & Chlorpheniramine & & 6.64 & \\
\hline \multirow[t]{3}{*}{ Sedation } & Control & 5.25 & 5.29 & 5.15 \\
\hline & Valerian-hops & 5.18 & 4.92 & 4.90 \\
\hline & Chlorpheniramine & 5.88 & 4.98 & 5.03 \\
\hline \multirow[t]{3}{*}{ Anxiety } & Control & 5.10 & 5.01 & 5.15 \\
\hline & Valerian-hops & 4.63 & 4.76 & 4.90 \\
\hline & Chlorpheniramine & 4.35 & 4.17 & 4.62 \\
\hline \multirow{3}{*}{$\begin{array}{l}\text { Sleep latency in } \\
\text { minutes }\end{array}$} & Control & & 71.83 & \\
\hline & Valerian-hops & & 64.11 & \\
\hline & Chlorpheniramine & & 36.08 & \\
\hline \multirow[t]{3}{*}{ Total hours slept } & Control & & 6.80 & \\
\hline & Valerian-hops & & 6.89 & \\
\hline & Chlorpheniramine & & 6.58 & \\
\hline
\end{tabular}

\section{Sedation at 1 hour, at awakening and at 24 hours}

As shown in Table 2, sedation was measured at 1 hour, after awakening and at 24 hours. One-way ANOVA was performed to examine the differences between the three groups in sedation at one hour, at awakening and at 24 hours.

One-way ANOVA showed that there were significant differences between the three groups in one hour $\mathrm{F}(2,189)=25.13, \mathrm{P}=0.001$, at awakening $\mathrm{F}(2,189)=6.51, \mathrm{P}=0.001$ but not in 24 hours $\mathrm{F}(2,189)$ $=1.82, \mathrm{P}=0.164$.

The post hoc test at one hour showed that the chlorpheniramine group had a significantly higher score compared to both placebo $(\mathrm{p}=0.001)$ and valerian-hops $(\mathrm{p}=0.001)$. However, there were no significant differences between valerian-hops and placebo groups at one hour.

Moreover, post hoc at awakenings in the placebo group had a significantly higher score compared to both valerian-hops ( $\mathrm{p}=$ $0.004)$ and chlorpheniramine groups $(p=0.036)$. However, there were no significant differences between the valerian-hops and chlorpheniramine group at awakenings.

\section{Anxiety at one hour, at awakening, and at 24 hours}

As shown in Table 2, anxiety was measured at 1 hour, at awakenings and at 24 hours. One-way ANOVA was utilized to examine the differences between the three groups' anxiety levels at one hour, at awakenings and at 24 hours.

One-way ANOVA showed that there were significant differences between the three groups in one hour $\mathrm{F}(2,189)=8.06, \mathrm{P}=0.001$, at awakenings $\mathrm{F}(2,189)=10.99, \mathrm{P}=0.001$ and at 24 hours $\mathrm{F}(2,189)$ $=5.63, \mathrm{P}=0.004$.

The post hoc test at one hour showed that the placebo group had significantly higher scores compared to both valerian-hops $(p=0.034)$ and chlorpheniramine groups $(p=0.001)$. However, 
there were no significant differences between the valerian-hops and chlorpheniramine groups at one hour.

Moreover, the post hoc test at awakenings in the placebo group had a significantly higher score compared to both the chlorpheniramine $(p=0.001)$ and valerian-hops groups $(p=0.007)$. However, there were no significant differences between the valerian and placebo groups at awakenings.

In addition, at 24 hours, the placebo group had a significantly higher score compared to chlorpheniramine $(p=0.004)$. However there were no significant differences between the Valerian and placebo groups, and between the valerian-hops and chlorpheniramine groups at 24 hours.

\section{Sleep latency and total hours slept}

As shown in Table 2, time to sleep and time of sleeping were reported after awakening. For the purpose of examining latency and actual differences in time to sleep in minutes and hours between the three groups, a one-way ANOVA was used. The test showed that there were significant difference between the three groups $\mathrm{F}(2,189)=13.11, \mathrm{p}=0.001$. The post hoc test showed that the placebo group had a significantly higher latency time compared to the chlorpheniramine group $(\mathrm{p}=0.001)$. In addition, the valerian-hops group had longer latency time compared to the chlorpheniramine group $(\mathrm{p}=0.001)$. There was no significant difference between placebo and valerian-hops groups. The results for the time of sleeping differences between the groups showed no significant differences.

\section{Participants' evaluation}

As shown in Table 3, each participant in the intervention group was asked to complete a treatment evaluation. There were three options: no improvement, slight improvement and marked improvement.

The Kruskal-Wallis test was used to examine the differences in patients' evaluations. The results indicate that there was a significant difference between them. Between the three groups (Chi square $=$ $12.45, \mathrm{p}=0.002)$ the higher mean rank was for the chlorpheniramine group (mean rank $=115.62$ ), followed by the valerian-hops group (mean rank $=96.28$ ), and the lowest was for the placebo group (mean rank $=82.86$ )

Table 3. Treatment evaluation

\begin{tabular}{|l|c|c|c|}
\hline \multirow{2}{*}{ Group } & \multicolumn{3}{|c|}{ Participant's self-evaluation } \\
\cline { 2 - 4 } & No improvement & $\begin{array}{c}\text { Slight } \\
\text { improvement }\end{array}$ & $\begin{array}{c}\text { Marked } \\
\text { improvement }\end{array}$ \\
\hline Control & 25 & 50 & 1 \\
\hline Valarian-hops & 22 & 28 & 15 \\
\hline Chlorpheniramine & 14 & 13 & 23 \\
\hline Total number & 61 & 91 & 39 \\
\hline
\end{tabular}

\section{Discussion}

This is the first study that has evaluated and compared the efficacy of valerian-hops and chlorpheniramine among insomniac refugees. Although our results demonstrated that neither valerianhops nor a chlorpheniramine single dose improved sleep, valerianhops combination demonstrated a significantly better sleep quality compared to chlorpheniramine.

The efficacy of valerian in improving sleep remains controversial. Previously, some studies have reported improved sleep outcomes with single or multiple doses of valerian ${ }^{19-21}$. On the contrary, our findings are consistent with recent research demonstrating nonsignificant improvement in sleep measures 22,23 .

This controversy could be explained by the different study sample, study design, extract type and valerian dose. All previous trials recruited patients with insomnia or healthy volunteers whereas in the current study all participants were recently displaced refugees.

No previous study compared valerian-hops with antihistamine single doses, however, a single study that compared valerian-hops with diphenhydramine demonstrated modest improvements on subjective sleep measures after 6 weeks ${ }^{24}$. Based on our observation that many insomniac subjects use a single dose of sedative/ anxiolytics. This is the first study comparing a single dose of valerianhops combination to antihistamine in improving subjective sleep measures among insomniac refugees.

The anxiolytic and sedative profiles of the two treatments can be explained as follows. Valerian-hops produces an "as needed" anxiolysis that is evident only after one hour compared to the extended anxiolysis and sedative effect of chlorpheniramine. This could be attributed to valerenic acid that is detectable in the serum only within the first hour of administration ${ }^{25}$.

This also explains the nonsignificant latency time reduction seen with valerian-hops compared to chlorpheniramine. Moreover, this favorable pharmacokinetic profile prevents residual effects manifested by cognitive and psychomotor side effects with sedating antihistamines.

The study has several weaknesses, as it relied only on subjective sleep measures. Furthermore, the randomization process was not according to standard procedure which could have led to possible bias. Also, the single dose design may not reflect the maximal potential benefit of valerian-hops. A cross-over design was not implemented. Furthermore, refugees are a highly anxiety-prone population, therefore, the results of this study may not be applied to insomniac patients from the normal population. In conclusion, insomnia represents a serious challenge for refugees. Valerianhops combination revealed better sleep quality than sedating antihistamine. Further studies are needed with multiple dosing design to reveal the potential benefit of this herb among refugees.

\section{Conclusion}

Our findings demonstrated that Valerian-hops combination may provide better sleep quality than antihistamines due to its sufficient anxiolytic effect. The study aimed to raise awareness for the need to study over the counter medicines. It may lead to better controlled randomized trials.

\section{Acknowledgments}

The authors would like to thank Caritas physicians Dr. Waleed Abu Al-Shar, Dr. Joseph Janho and Dr. Nader Hijazeen. Special thanks for Dr. Malak Tayfur for her help. Also special thanks for Beitlahem Drug store. The corresponding author would like to thank Rafael and Dr. Moscati for their continuous work.

\section{Research funding}

This study was funded by the American University of Madaba, Madaba, Jordan.

\section{Disclosure}

The authors declare no conflict of interest.

\section{References}

1. Roth T. Insomnia: definition, prevalence, etiology, and consequences. J Clin Sleep Med. 2007;3(5 Suppl):S7-10.

2. Léger D, Partinen M, Hirshkowitz M, Chokroverty S, Hedner J; EQUINOX (Evaluation of daytime QUality Impairment by Nocturnal awakenings in Outpatient's eXperience) Survey Investigators. Characteristics of insomnia in a primary care setting: EQUINOX survey of 5293 insomniacs from 10 countries. Sleep Med. 2010;11(10):987-98. 
3. Unbehaun T, Spiegelhalder K, Hirscher V, Riemann D. Management of insomnia: update and new approaches. Nat Sci Sleep. 2010;2:127-38.

4. Pigeon WR. Diagnosis, prevalence, pathways, consequences \& treatment of insomnia. Indian J Med Res. 2010;131:321-32.

5. Bhugra D. Migration and mental health. Acta Psychiatr Scand. 2004;109(4):243-58.

6. Lee YJ, Jun JY, Lee YJ, Park J, Kim S, Lee SH, et al. Insomnia in North Korean Refugees: Association with Depression and Post-Traumatic Stress Symptoms. Psychiatry Investig. 2016;13(1):67-73.

7. GAMMOUH, Omar Salem, et al. Peer Reviewed: Chronic Diseases, Lack of Medications, and Depression Among Syrian Refugees in Jordan, 2013-2014. Preventing Chronic Disease, 2015, 12.

8. Basishvili T, Eliozishvili M, Maisuradze L, Lortkipanidze N, Nachkebia $\mathrm{N}$, Oniani $\mathrm{T}$, et al. Insomnia in a displaced population is related to war-associated remembered stress. Stress Health. 2012;28(3):186-92.

9. Aranko K, Mattila MJ, Seppälä TO. Development of tolerance and cross-tolerance to the psychomotor actions of lorazepam and diazepam in man. Br J Clin Pharmacol. 1983;15(5):545-52.

10. Izumi N, Mizuguchi H, Umehara H, Ogino S, Fukui H. Evaluation of efficacy and sedative profiles of $\mathrm{H}(1)$ antihistamines by large-scale surveillance using the visual analogue scale (VAS). Allergol Int. 2008;57(3):257-63.

11. Rosenberg HC, Chiu TH. Time course for development of benzodiazepine tolerance and physical dependence. Neurosci Biobehav Rev. 1985 Spring;9(1):123-31.

12. Murti K, Kaushik M, Sangwan Y, Kaushik A. Pharmacological properties of Valeriana officinalis - A review. Pharmacologyonline. 2011;3:641-6.

13. Patočka J, Jakl J. Biomedically relevant chemical constituents of Valeriana officinalis. J Appl Biomed. 2010;8:11-8.

14. Morin CM, Koetter U, Bastien C, Ware JC, Wooten V. Valerian-hops combination and diphenhydramine for treating insomnia: a randomized placebo-controlled clinical trial. Sleep. 2005;28(11):1465-71.

15. Morin CM, et al. Insomnia. John Wiley \& Sons, Inc., 1993.
16. Cornu C, Remontet L, Noel-Baron F, Nicolas A, Feugier-Favier N, Roy P, et al. A dietary supplement to improve the quality of sleep: a randomized placebo controlled trial. BMC Complement Altern Med. 2010;10:29.

17. Suleiman $\mathrm{KH}$, Yates BC. Translating the insomnia severity index into Arabic. J Nurs Scholarsh. 2011;43(1):49-53.

18. Tarrasch R, Laudon M, Zisapel N. Cross-cultural validation of the Leeds sleep evaluation questionnaire (LSEQ) in insomnia patients. Hum Psychopharmacol. 2003;18(8):603-10.

19. Balderer G, Borbély AA. Effect of valerian on human sleep. Psychopharmacology (Berl). 1985;87(4):406-9.

20. Donath F, Quispe S, Diefenbach K, Maurer A, Fietze I, Roots I. Critical evaluation of the effect of valerian extract on sleep structure and sleep quality. Pharmacopsychiatry. 2000;33(2):47-53.

21. Ross SM. Sleep disorders: a single dose administration of valerian/hops fluid extract (dormeasan) is found to be effective in improving sleep. Holist Nurs Pract. 2009;23(4):253-6.

22. Taibi DM, Vitiello MV, Barsness S, Elmer GW, Anderson GD, Landis CA. A randomized clinical trial of valerian fails to improve self-reported, polysomnographic, and actigraphic sleep in older women with insomnia. Sleep Med. 2009;10(3):319-28.

23. Diaper A, Hindmarch I. A double-blind, placebo-controlled investigation of the effects of two doses of a valerian preparation on the sleep, cognitive and psychomotor function of sleep-disturbed older adults. Phytother Res. 2004;18(10):831-6.

24. Anderson GD, Elmer GW, Kantor ED, Templeton IE, Vitiello MV. Pharmacokinetics of valerenic acid after administration of valerian in healthy subjects. Phytother Res. 2005;19(9):801-3.

25. Kamei H, Isaji A, Noda Y, Ishikawa K, Senzaki K, Yamada K, et al. Effects of single therapeutic doses of promethazine, fexofenadine and olopatadine on psychomotor function and histamine-induced wheal- and flare-responses: a randomized double-blind, placebo-controlled study in healthy volunteers. Arch Dermatol Res. 2012;304(4):263-72. 